\title{
Research on hardware virtual simulation based on Multisim
}

\author{
Yurong Guan ${ }^{1,}$ a , Fen Zhou ${ }^{2, b}$ \\ ${ }^{1}$ Huanggang Normal University, Huangzhou, Hubei, China \\ ${ }^{2}$ Huanggang Normal University, Huangzhou, Hubei, China \\ ajsjgyr@hgnu.edu.cn, bjsjzhoufen@hgnu.edu.cn
}

\begin{abstract}
Keywords: Multisim, computer hardware, virtual simulation
Abstract. Computer hardware course is abstract, Multisim simulation software will be integrated into the computer hardware course teaching, promote the teaching to the development of information technology through the simulation play the role of technology, brings to the teachers and students professional and comprehensive virtual experiment environment. This paper first introduces the basic situation of Multisim software platform, combined with the practice of the current computer hardware curriculum system of computer composition principle of this course, analyzes the composition of the functional units of the computer virtual simulation design and implementation method, discusses the important role of the virtual simulation technology platform, a comprehensive system of value of the virtual simulation the hardware based on Multisim.
\end{abstract}

\section{Introduction}

Virtual experiment is one of the most popular technologies at home and abroad, many high-tech, high investment and high risk project, using the virtual experiment technology, such as nuclear power, aerospace, astrophysics, climate, ocean currents, crustal movement can be simulated, of course, is mainly used for product development and experimental teaching in civilian areas, including electronic design automation is the development and application of the most mature, can be realized on the computer, without the need to build the actual circuit, there are quite a number of commercial products, such as Spice, Pspice, EwB, MAx+P1usII, Systemview, QuartusII, Multisim etc..

\section{The basic overview}

Multisim is a simulation tool based interactive image technologies and Electronics Workbench launched to Windows, for board level analog or digital circuit board design, it contains graphic input, hardware circuit principle diagram description language input, with extensive simulation analysis.

A desktop running as Windows electronic design tools, Multisim [1] is a complete integrated design environment, the interface as an experimental electronic workbench, drawing components required by the circuit simulation and the required test equipment can directly drag it to the screen, the mouse can wire connecting them, software instrument the control panel and the operation mode are similar with real measured data, waveform and characteristic curve as seen in the real instrument; provides more than 17000 kinds of elements in the mainstream of the world element provider, but also convenient to edit and modify the components of various parameters, can use the model and code generator mode to create the model and other functions, to create their own the components, using SPICE3F5 and Xspice kernel as the simulation engine [2], with the workbench by Electronic Enhance the design function, optimize the simulation performance of digital and mixed mode, and provide 22 kinds of virtual instruments to measure the action of the circuit.

There are real elements and virtual components in Multisim [3]. The fundamental difference between them lies in: one is the model, parameters and actual values and components of the packages are corresponding components, using this device in the design, not only can make the design of the simulation corresponds well with the actual situation, the design can also be directly derived design the Ultiboard to PCB. The parameter value of the other device is the typical value of this kind of device, which is not corresponding to the actual device. The user can change the parameter value of 
the device model according to the need, and can only be used for simulation. Such devices are called virtual devices.

\section{Simulation and design of functional components of computer}

At present, composition teaching experiment principle has three major drawbacks of major colleges and universities, rarely from the hardware description language, and began to understand, or gate logic circuit, on the basis of in-depth study and understand the structure of CPU, and realize yourself.

On the platform of pure hardware experiment, most students are bound in the process of inserting thread. After the plug line is finished, the mechanical toggle corresponding switch is not clear about the result of the right or wrong.

Without independent experiments, students can only perform functional verification on existing systems because the system is solidified.

The experimental platform is mostly micro program control mode, so that students can not fully understand the current large-scale and large computer system controller.

The principle component of the computer [4] such as large scale hardware systems practice as an example, in order to deepen the basic principle and the concept of students' understanding of the computer, students need to make up a computer system for analysis, design and implementation of the large scale integrated circuit device. First of all, students are required to design several functional components of the computer system, so that they can apply the theoretical knowledge in the design process to the actual design and debugging work.

In the virtual simulation experiment of the functional components of the computer system, the main design is as follows:

Arithmetic function unit: the design of function unit of 8 bits or more, including the realization of various arithmetic and logic functions.

General register set: the design and implementation of each register read and write function in the general register set.

Memory function components: the design of memory capacity components of corresponding capacity, the experiment of memory word extension, bit extension and word bit simultaneous expansion.

Program counter: control instruction address for increasing and transfer procedures, counters design and implementation, storage of the next instruction in the main memory address.

Clock generator: the design and implementation of timing generator, providing all the working cycle signals, timing signals and work pulse signals needed by the model machine.

Data path: data path design and implementation of instruction execution.

The above features need to be separate designed, compiler and simulation, with the waveform of the simulation results to verify the correctness of each components of the final design, the encapsulation of each functional module design, complete the debugging.

\section{The realization method of virtual technology}

The virtual operation of Multisim technology to computer hardware [5] is simple and convenient. It can arouse the interest of study and link up the theoretical knowledge and practice.

To create a student learning situation of inquiry, students can independently operate the experiment, to stimulate interest in learning, to provide interactive performance of multidimensional learning environment, fully mobilize the enthusiasm of students in the process of learning habits, learning to enable students to change from passive to active exploration.

The use of Multisim technology to simulate the use of hardware chip, and through the analysis of the characteristics of open experimental teaching of simulation software requirements, the virtual simulation technology is applied to the design and development of computer hardware, helps to broaden the knowledge. 
Pay attention to the direction of cutting-edge technology, and use virtual cases to strengthen the teaching effect. Virtual simulation technology can play a positive role in each link of the whole teaching system, and constantly improve the quality of teaching.

\section{Application analysis of simulation platform}

Using visual simulation software, students can realize the circuit design, component selection, layout, wiring, programming, data set, operation circuit, observe the operation status and a series of operations, the whole hardware circuit structure, operation process and circuit development process will have a better understanding, can also be developed the expansion of software components.

Provide innovative experimental platform. For example, the composition principle of computer in virtual laboratory, experimental assembly can undertake CPU, and this experiment in traditional hardware experiment platform, due to lack of performance support components and the limitation of the chip can't be achieved.

Provide practical platform for algorithms in related courses. In component-based virtual laboratory system, students can experiment only with components of the core algorithm, and the input and output components will be provided by the system to simplify the design.

Provide extracurricular optional experiment platform. The virtual lab system can be accessed at anytime and anywhere through the network without having to be bound by site or time.

Providing secondary platforms. In the process of teaching, teachers can access the virtual laboratory system through Internet to do relevant experiments, which can greatly enrich the classroom teaching and improve the teaching effect.

Provide a lossless safety platform. The hardware experiment software, can be convenient and bold to carry out various attempts, without worrying about the impact of hardware loss.

Computer hardware experiment simulation platform to the teaching practice, is a simulation software of computer hardware, it can simulate the computer hardware circuit behavior, the completion of the simulation of computer hardware experiment task.

The choice of simulation software instead of dedicated hardware experimental equipment, its starting point is to have more comprehensive functions, more convenient operation, lower cost.

As a virtual experiment platform of computer hardware experiment, it should include three parts: virtual component, simulation of experiment system and visual operation interface.

\section{The main problems to be solved}

Multisim combines intuitive capture and powerful simulation, enabling rapid, easy and efficient design and verification of circuits. With Multisim simulation, you can create a complete circuit component library immediately, and using the circuit simulator to imitate behavior, with professional analysis and virtual instrument, can quickly verify early in the design process of circuit design, so as to shorten the modeling cycle.

Through the intuitive circuit diagram to capture the environment, easy to design hardware chip circuit.

Quickly understand circuit behavior through interactive reality.

Understanding basic chip design features by means of advanced circuit analysis.

Seamlessly integrate circuit design and virtual testing through a tool chain.

The objectives of the study are as follows:

Virtual analog memory circuit implementation.

The realization of virtual simulation controller circuit.

On the basis of mastering the unit circuit, it is further realized by logical composition, and a basic model computer is constructed in a systematic way.

Improve students' system architecture and modular design ideas. 


\section{Conclusions}

By means of Multisim and virtual instrument technology, the circuit schematic diagram can be built interactively, and the circuit can be simulated. Multisim refines the complex content of SPICE simulation, so no need to know deeply the SPICE technology can quickly capture, simulation and analysis of the new design, which makes it more suitable for the virtual simulation experiment of computer hardware courses. The use of virtual simulation software and traditional teaching methods in classroom teaching in the combination of the circuit principle through the simulation software in a dynamic way is presented, and the simulation instrument convenient access to experimental data, visually help students establish the concept of abstraction, also guide the students in the class by using the simulation software to ponder and analyze at the same time, the cultivation of students' innovation ability.

\section{Acknowledgements}

I thank reviewers for constructive reviews and suggestions that improved the quality of this paper. This essay is the fruit of our several months' consisting work. This work was supported by the research on virtual hardware simulation based on Multisim of experimental teaching research center in 2017 of experimental teaching center of Huanggang Normal University.

\section{References}

[1] Liu Hui, Huang Ying. Application of Multisim in the teaching reform of computer hardware course, science and technology, 2013.10

[2] Yi Xiaolin, Zhao Lei, Zhou Wenke, Zhang Haibin. Research on Virtualization course of computer system hardware course based on EDA platform, Chinese university education, 2005.7

[3] Lei Xiangdong, Lei Zhenyang, Yuan Xiaoli, Huang Dongjun. Research on strengthening the construction of computer hardware curriculum system and improving teaching quality, Education and teaching forum, 2012, twenty-fifth

[4] Nie Dian. Application of Multisim 10 computer simulation in electronic circuit design [M]. Beijing: electronics industry press, 2009.

[5] Huang Peigen, Ren Qing Bao. Multisim10 computer virtual simulation laboratory [M]. Beijing: electronics industry press, 2008 